\title{
Morfologia e Estereologia do Miocárdio em Ratos Hipertensos. Correlação com o Tempo de Inibição da Síntese do Óxido Nítrico
}

Leila Maria Meirelles Pereira, Glória Maria Moraes Vianna, Carlos A lberto Mandarim-de-Lacerda

Rio de Janeiro, RJ

Objetivo - Estudar mudanças estruturais do miocárdio decorrentes do tempo de inibição da síntese do óxido nítrico.

Métodos - Estudaram-se 4 grupos de 10 ratos: controle e L-Name (50mg/kg/dia) com 25 e 40 dias de experimentação. Os animais foram sacrificados e os corações processados tecnicamente para estudo em microscopia de luz, onde cortes corados pelo picro-sirius foram estudados com e sem luz polarizada para análise do colágeno do interstício cardíaco. Determinaram-se densidades de volume de miócitos ( $V v[m])$ e interstício cardíaco (Vv[int]), densidade numérica de miócitos $(\mathrm{Nv}[\mathrm{m}])$ e área transversal dos miócitos $(A[m])$.

Resultados - Compararam-se os animais L-Name com os respectivos controles. Nos L-Name a pressão da cauda aumentou 74,5 e 90,2\% aos 25 e 40 dias, respectivamente. O peso cardíaco aumentou $50 \%$ e $28 \%$ aos 25 e 40 dias, respectivamente. $O$ miocárdio dos animais L-Name apresentou hipertrofia de miócitos com aumento da $A(m)$, fibrose perivascular e intersticial, espessamento das túnicas íntima e média das artérias intramiocárdicas. Aos 40 dias diminuíram $V v(m)$ e $N v(m)$ e aumentou $V v$ (int).

Conclusão - Inibição da síntese do óxido nítrico provoca modificações miocárdicas que progridem como tempo de administração de L-Name. A estereologia é uma metodologia útil para determinar e avaliar as modificações miocárdicas decorrentes deste modelo experimental de hipertensão arterial.

Palavras-chave: óxido nítrico, miocárdico, hipertensão, estereologia

\section{Morphology and Stereology of the Myocardium in Hypertensive Rats. Correlation with the Time of Nitric Oxide Synthesis Inhibition}

Purpose - To study structural changes of the myocardium in relation to the time of nitric oxide synthesis inhibition.

Methods - Four groups of 10 rats each were studied: 2 control groups and 2 groups with administration of LName (50mg/kg/day), one during 25 days and the other during 40 days. The animals were then sacrificed and the hearts were prepared for study in light microscopy, where sections stained by picro-sirius were studied with and without polarized light for analysis of the cardiac interstitium collagen. Volume densities of myocytes $(V v[m])$ and interstitium ( $V v[$ int $])$, the numerical density of myocytes $(\mathrm{Nv}[\mathrm{m}])$ and the mean cross-sectional area of myocytes were $(A[m])$ also determined.

Results - The L-Name animals were compared with the respective controls. In the L-Name rats, the tail arterial pressure increased 74.5 and $90.2 \%$ in the 25 days group and in the 40 days group, respectively. The heart weight increased $50 \%$ in the 25 days group and $28.6 \%$ in the 40 days group. The myocardium of the L-Name animals presented myocyte hypertrophy with increased $A(m)$, perivascular and interstitial fibrosis, thickness of the tunica intima and tunica media of the intramyocardial arteries. In the 40 days group the L-Name animals had decreased $V v(m)$ and $N v(m)$ and increased $V v$ (int).

Conclusion - Inhibition of nitric oxide synthesis provokes myocardial changes that progress with the time of L-Name administration. The stereology is useful to determine and to evaluate the myocardial changes in this model of arterial hypertension.

Key-word: nitric oxide, myocardium, hypertension, stereology

Arq Bras Cardiol, volume 70 (nº 6), 397-402, 1998

Instituto de Biologia Roberto Alcântara Gomes - Laboratório de Morfometria e Morfologia Cardiovascular - UERJ

Correspondência: Leila Maria Meirelles Pereira - Centro Biomédico - Av. 28 de Setembro, 87 (fundos) - 20551-030 - Rio de Janeiro, RJ

Recebido para publicação em 26/1/98

Aceito em 1/4/98
A hipertrofia miocárdica é a tentativa de adaptação do coração à sobrecarga de trabalho, evoluindo para falência cardíaca quando o processo de adaptação é esgotado ${ }^{1}$. No conjunto da hipertrofia miocárdica há alterações vas- 
culares, com o aumento da espessura da parede dos vasos da microcirculação coronária com conseqüente redução do lume vascular e aumento da biossíntese de colágeno na parede vascular, e mudanças na estrutura do miocárdio, como o remodelamento intersticial, com acúmulo de colágeno fibrilar que causa enrijecimento miocárdico ${ }^{2-4}$. A ação do sistema renina-angiotensina-aldosterona também promove acúmulo miocárdico de colágeno tipos I e III aumentando a disfunção ventricular sistólica e diastólica. Em conseqüência, o remodelamento estrutural da matriz colágena do coração faz progredir a disfunção ventricular até a falência cardíaca congestiva ${ }^{5,6}$.

Miofibroblastos atuam na matriz extracelular do coração com atividade contráctil e metabólica. São células transformadas fenotipicamente em fibroblastos que expressam $\alpha$-actina de músculo liso e são responsáveis pelo turnover do colágeno nos locais de reparação miocárdica. Hoje, sabe-se que a síntese "de novo" de angiotensina II pelos miofibroblastos nos locais de reparação miocárdica tem importante função autócrina e parácrina. Assim, as formas de fibrose miocárdica regressiva, persistente e progressiva estão relacionadas com a ação dos miofibroblastos e os sinais que eles geram ${ }^{7}$.

A inibição da enzima sintetizadora do óxido nítrico promove hipertensão arterial (HA) de modo rápido e permanente, caracterizando-se por um aumento na resistência periférica total dos vasos sangüíneos ${ }^{8}$. O L-Name $\left(\mathrm{N}^{\mathrm{G}}\right.$-nitro-metilester-L-arginina), cuja ação está ligada à competição com o aminoácido L-arginina (seu análogo enantiométrico) na via de produção do óxido nítrico, leva a inibição da síntese deste último com conseqüente vasoconstrição e elevação dos níveis de pressão arterial (PA) ${ }^{9-11}$. A deficiência crônica de óxido nítrico causa HA acompanhada de hipertrofia cardíaca $^{12-15}$. Mas, ainda há controvérsia quanto a exata interpretação dos alterações miocárdicas causadas por este modelo de HA ${ }^{12,16,17}$. Em animais submetidos ao uso de L-Name, a hipertrofia miocárdica não é proporcional aos níveis tensionais e as alterações miocárdicas intersticiais são mais intensas que às apresentadas em outros modelos de HA (Goldblatt II, por exemplo) ${ }^{4,18}$.

O presente estudo morfológico e quantitativo em ratos hipertensos pela administração de L-Name tem o objetivo de analisar as mudanças estruturais do miocárdio, particularmente os ramos intramiocárdicos das artérias coronárias.

\section{Métodos}

Foram utilizados 40 rattus norvegicus, variedade Wistar, adultos jovens, machos, não consangüíneos, inicialmente com peso corporal entre 200 e $280 \mathrm{~g}$ e PA da cauda inferior a 130mmHg. Antes de iniciar o estudo os animais foram mantidos, por duas semanas, em observação. A PA foi verificada a cada semana, caracterizando a normotensão. A partir daí, foram divididos em quatro grupos de 10 ratos, mantidos em caixas plásticas adequadas; a) controle 25 dias; b) controle 40 dias; c) L-Name 25 dias; d) L-Name 40 dias.

Os animais dos grupos controles receberam água e ra- ção padrão Purina ${ }^{\circledR}$ ad libitum durante 25 e 40 dias, respectivamente. Os animais dos grupos experimentais (grupos L25 e L40), receberam L-Name (hidrocloreto de $\mathrm{N}^{\mathrm{G}}$-nitro-Larginina-metil, ester, Sigma Co, St. Louis, lote 44H0102) por 25 ou 40 dias, respectivamente, na dose de $50 \mathrm{mg} / \mathrm{kg} / \mathrm{dia}$, dissolvidos na água do bebedouro.

No $26^{\circ}$ e no $41^{\circ}$ dias de experimentação os animais dos grupos L-Name e seus respectivos controles foram anestesiados por inalação de éter etílico e sacrificados com injeção intracardíaca de $\mathrm{KC} 1$ a 10\% (promove parada cardíaca em diástole). Os corações foram retirados, pesados em balança analítica segundo o método de Scherle ${ }^{19}$ suspensos por um fio e imersos em solução fisiológica num recipiente sobre o prato da balança sem tocar as paredes do recipiente. Depois, foram imediatamente fixados em solução de formol $10 \%$ tamponado $(\mathrm{pH} 7,2)$ por $48 \mathrm{~h}$.

Fragmentos do miocárdio da parede ventricular esquerda e septo interventricular foram obtidos conforme o método de clivagem denominado orthotrip ${ }^{20}$, obtendo-se cortes "aleatórios e uniformemente isotrópicos", necessários para o estudo estereológico ${ }^{21}$. Processou-se o material tecnicamente com inclusão em parafina e microtomia com $5 \mu \mathrm{m}$ de espessura. Os cortes foram corados com picro-sirius e tricrômico de van Gieson.

As densidades de volume e de número dos miócitos cardíacos foram determinados. Os cálculos estereológicos foram realizados em 15 campos microscópicos aleatórios por coração ${ }^{22-24}$. Usou-se objetiva de imersão em óleo e sistema de vídeo-microscopio composto por microscópio Leica ${ }^{\circledR}$ modelo DMRBE acoplado a uma câmara de vídeo Kappa ${ }^{\circledR}$ CF $15 / 5$ e um monitor Sony ${ }^{\circledR}$. O sistema-teste M42 foi sobreposto a tela do monitor e calibrado com micrômetro-objeto $1 \mathrm{~mm} / 100$ Leitz®. Os parâmetros estereológicos foram determinados de acordo com a metodologia previamente estabelecida ${ }^{14,15,18}$.

Além da estatística descritiva, as diferenças estereológicas, comparando os grupos de animais controle com L-Name nos dias 25 e 40 foram testadas pelo teste não paramétrico Mann-Whitney com índice de significância de $0,05^{25}$.

\section{Resultados}

Nos animais que receberam L-Name a PA da cauda foi significativamente maior que nos respectivos controles. Foi $74,5 \%$ mais elevada com 25 dias e $90,2 \%$ com 40 dias. Comparando os animais hipertensos com os respectivos controles de mesma idade, o peso cardíaco foi maior $50 \%$ com 25 dias e 28,6\% com 40 dias de experimentação (tab. I e II).

Com 25 dias, e mais intensamente com 40 dias de HA, os miócitos cardíacos estiveram hipertrofiados com acentuado aumento de miofibrilas citoplasmáticas. Nestes animais hipertensos, o miocárdio, além das áreas de fibrose, mostrou áreas com lesões isquêmicas apresentando infiltrado inflamatório e fibrose cicatricial (de substituição). Isto, provavelmente, deveu-se a necrose de miócitos nestas áreas onde também observou-se espessamento da túnica média dos vasos intramiocárdicos. 


\begin{tabular}{|c|c|c|c|c|c|c|}
\hline Grupo & $\begin{array}{l}\mathrm{Nv}(\mathrm{m}) \\
1 / \mathrm{mm}^{3}\end{array}$ & $\begin{array}{r}\mathrm{Vv}(\mathrm{m}) \\
\%\end{array}$ & $\begin{array}{r}\mathrm{Vv} \text { (int) } \\
\%\end{array}$ & $\begin{array}{r}\mathrm{A}(\mathrm{m}) \\
\mu \mathrm{m}^{2}\end{array}$ & $\begin{array}{r}\mathrm{PA} \\
\mathrm{mmHg}\end{array}$ & $\begin{array}{r}\text { Peso } \\
(\mathrm{g})\end{array}$ \\
\hline \multicolumn{7}{|l|}{ Controle } \\
\hline Mediana & 45565,5 & 56,3 & 44,7 & 173,0 & 107,2 & 0,6 \\
\hline \multirow[t]{3}{*}{ IC $95 \%$} & 37416,7 & 50,31 & 38,4 & 158,7 & 104,5 & 0,5 \\
\hline & $\mathrm{a}$ & $\mathrm{a}$ & $\mathrm{a}$ & $\mathrm{a}$ & a & $\mathrm{a}$ \\
\hline & 52846,4 & 60,82 & 50,4 & 188,3 & 109,7 & 0,7 \\
\hline CE\% & 6,1 & 3,4 & 3,2 & 3,1 & 2,1 & 5,1 \\
\hline \multicolumn{7}{|l|}{ L-Name } \\
\hline Mediana & 42310,8 & 54,1 & 46,6 & 363,3 & 187,1 & 0,9 \\
\hline \multirow[t]{3}{*}{ IC 95\% } & 40312,1 & 51,0 & 40,6 & 349,9 & 175,2 & 0,8 \\
\hline & $\mathrm{a}$ & $\mathrm{a}$ & $\mathrm{a}$ & $\mathrm{a}$ & a & $\mathrm{a}$ \\
\hline & 47781,2 & 58,4 & 50,0 & 383,9 & 190,3 & 1,0 \\
\hline CE\% & 3,9 & 3,0 & 3,8 & 2,1 & 3,5 & 6,6 \\
\hline $\mathrm{p}$ & NS & NS & NS & 0,002 & 0,001 & 0,002 \\
\hline
\end{tabular}

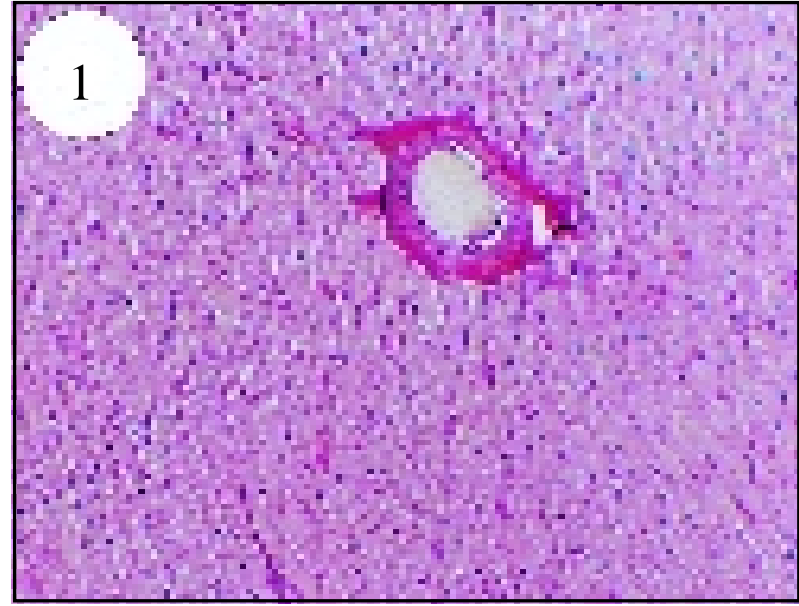

Fig. 1 - Fotomicrografia de corte transverso do miocárdio, passando por artéria de pequeno calibre, de animal do grupo controle com 40 dias (picro-sirius, $\mathrm{x} 140$ ).



Fig. 2 - Fotomicrografia de miocárdio de animal L-Name 40 dias onde observa-se área de lesão isquêmica recente com infiltrado inflatório (van Gieson, x 280).

Na figura 1, observa-se o miocárdio normal de animais do grupo controle 40 dias e nas figuras 2 a 4 o miocárdio dos animais hipertensos pelo uso do L-Name. Identificam-se áreas de lesão isquêmica aguda do miocárdio com a presença de células inflamatórias (fig. 2), áreas com extensa fibrose intersticial, predominantemente no endomísio e no peri-
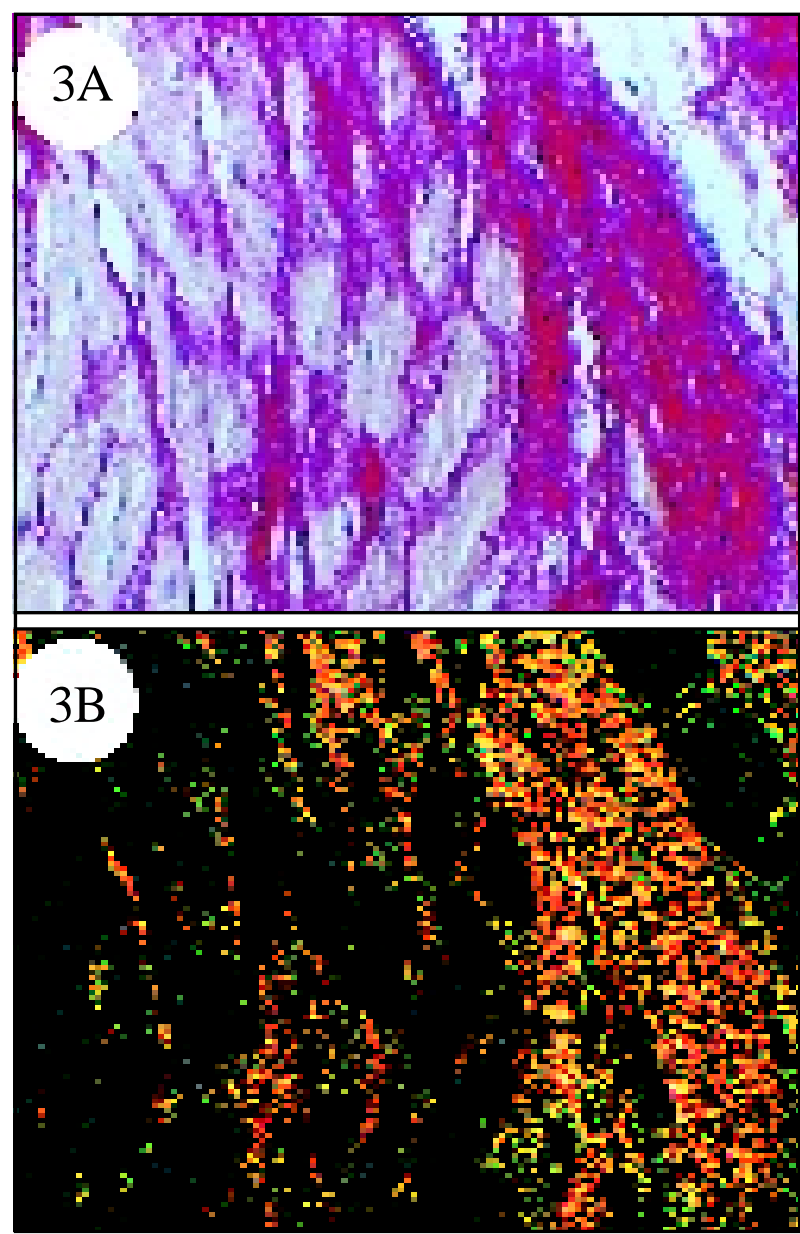

Fig. 3 - A) Fotomicrografias de miocárdio de animal L-Name 40 dias onde observa-se área de extensa fibrose intersticial. Em campo claro vê-se que o tecido conjuntivo (vermelho) substituiu os miócitos que necrosaram, os restantes estão hipertrofiados; B) em campo escuro (luz polarizada), vê-se o mesmo campo da figura anterior, porém a birrefringência do colágeno mostra grande quantidade de fibras colágenas vermelhoamarelo (provavelmente tipo I) e menor quantidade de fibras colágenas verdes (provavelmente tipo III) (picro-sirius, x 280).

mísio, caracterizadas pela cor vermelha na coloração pelo picro-sirius (fig. 3A) e, sob luz polarizada, por aumento na concentração de fibras colágenas de cor vermelho alaranjada (provavelmente colágeno tipo I) e, em menor quantida- 


\begin{tabular}{|c|c|c|c|c|c|c|}
\hline Grupo & $\begin{array}{l}\mathrm{Nv}(\mathrm{m}) \\
1 / \mathrm{mm}^{3}\end{array}$ & $\begin{array}{r}\mathrm{Vv}(\mathrm{m}) \\
\%\end{array}$ & $\begin{array}{r}\text { Vv(int) } \\
\%\end{array}$ & $\begin{array}{r}\mathrm{A}(\mathrm{m}) \\
\mu \mathrm{m}^{2}\end{array}$ & $\begin{array}{r}\mathrm{PA} \\
\mathrm{mmHg}\end{array}$ & $\begin{array}{r}\text { Peso } \\
(\mathrm{g})\end{array}$ \\
\hline \multicolumn{7}{|l|}{ Controle } \\
\hline Mediana & 65093,6 & 53,0 & 47,40 & 358,0 & 103,8 & 0,6 \\
\hline \multirow[t]{3}{*}{ IC $95 \%$} & 56967,0 & 50,3 & 40,94 & 309,1 & 99,5 & 0,6 \\
\hline & $\mathrm{a}$ & $\mathrm{a}$ & $\mathrm{a}$ & $\mathrm{a}$ & a & a \\
\hline & 77993,68 & 57,8 & 50,99 & 392,5 & 107,1 & 0,8 \\
\hline $\mathrm{CE} \%$ & 7,1 & 3,1 & 3,9 & 5,2 & 3,4 & 5,2 \\
\hline \multicolumn{7}{|l|}{ L-Name } \\
\hline Mediana & 52074,9 & 46,7 & 53,2 & 390,9 & 204,8 & 0,9 \\
\hline \multirow[t]{3}{*}{ IC $95 \%$} & 44258,3 & 43,3 & 48,4 & 368,1 & 188,4 & 0,8 \\
\hline & $\mathrm{a}$ & $\mathrm{a}$ & a & a & $\mathrm{a}$ & $\mathrm{a}$ \\
\hline & 59023,5 & 51,1 & 57,2 & 442,1 & 209,2 & 1,0 \\
\hline CE\% & 6,3 & 3,7 & 3,4 & 4,2 & 5,1 & 4,5 \\
\hline $\mathrm{p}$ & 0,02 & 0,01 & 0,006 & 0,05 & 0,0005 & 0,001 \\
\hline
\end{tabular}

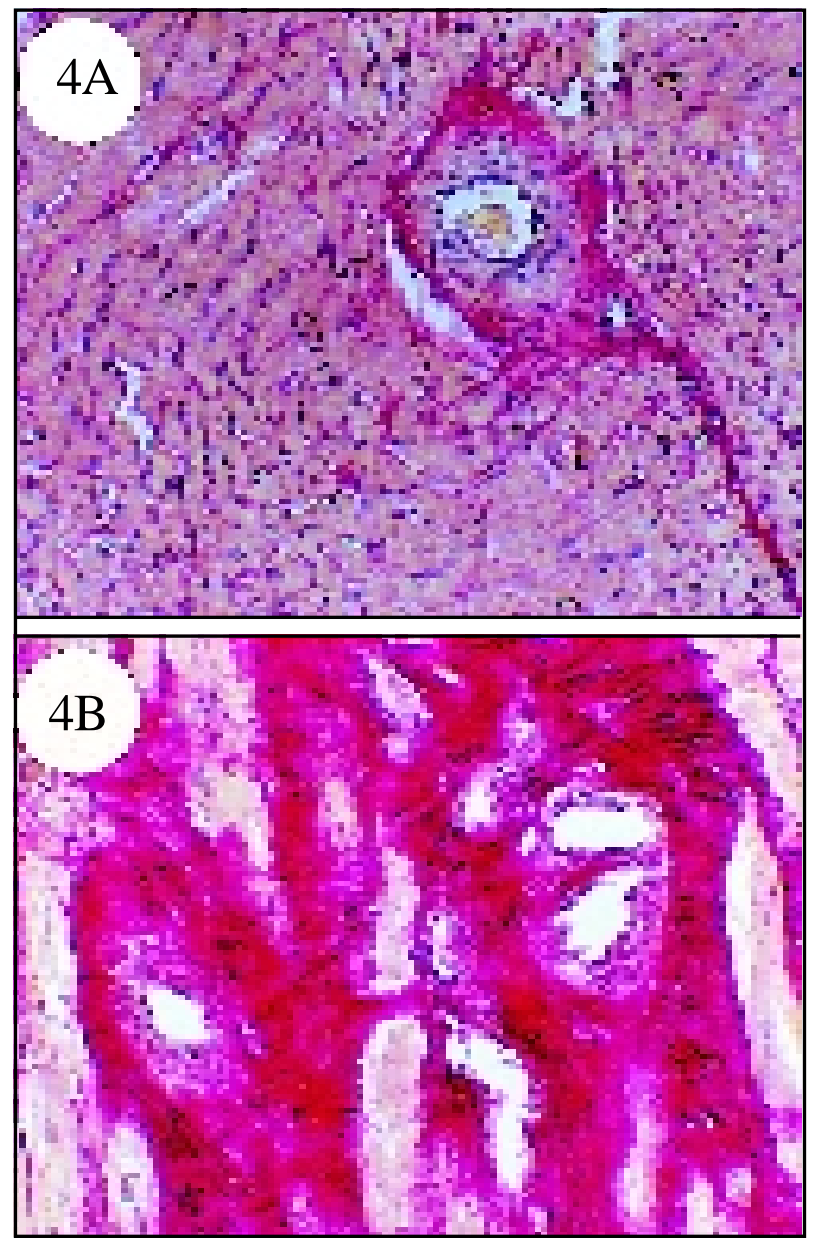

Fig. 4 - A) Fotomicrografias de corte transverso do miocárdio de animal L-Name aos 25 e aos 40 dias. Aos 25 dias há um pouco de fibrose perivascular e espessamento da túnica média do vaso; B) acentuado aos 40 dias (picro-sirius, x 280).

de, de fibras colágenas de cor verde (provavelmente colágeno tipo III) (fig. 3B). Nos animais hipertensos, tanto aos 25 quanto aos 40 dias, também observou-se fibrose perivascular com espessamento da túnica média nas artérias coronárias intramiocárdicas de menor calibre, com aumento no conteúdo de colágeno, principalmente o do tipo I.
Nos animais hipertensos, houve progressão na intensidade da fibrose intersticial e perivascular de 25 a 40 dias, com espessamento da túnica média dos vasos (fig. 4A e B).

Os resultados estereológicos encontram-se nas tabelas I e II. Comparando os animais hipertensos com seus respectivos controles de mesma idade, a densidade de volume dos miócitos ( $\mathrm{Vv}[\mathrm{m}])$ não apresentou diferença aos 25 dias, mas diminuiu 12,6\% aos 40 dias. Da mesma forma, a densidade de volume do interstício cardíaco (Vv[int]) não apresentou diferença aos 25 dias, mas aumentou 14,8\% aos 40 dias. A densidade numérica de miócitos $(\mathrm{Nv}[\mathrm{m}])$ também não apresentou diferença entre os animais hipertensos e os respectivos controles aos 25 dias, mas foi $23,5 \%$ menor nos animais hipertensos aos 40 dias. A área transversal média dos miócitos $(\mathrm{A}[\mathrm{m}])$ foi $111,4 \%$ maior aos 25 dias e apenas $15,5 \%$ maior aos 40 dias.

\section{Discussão}

O aumento da PA nos animais que receberam L-Name, no presente estudo, foi superior ao relatado na literatura, utilizando protocolo semelhante ao nosso ${ }^{10,26}$. Contudo, as alterações morfológicas observadas aqui assemelham-se às descritas na literatura para a inibição da síntese do óxido nítrico ${ }^{4,14-15,27}$.

Na sobrecarga de trabalho devido ao aumento da PA há aumento no tamanho do coração sem proliferação celular ${ }^{28}$. A parede dos ventrículos do coração de mamíferos apresenta extraordinária hipertrofia dos miócitos e hiperplasia das células intersticiais ${ }^{29}$. O remodelamento estrutural do miocárdio na HA ocorre por alteração do colágeno decorrente do crescimento de fibroblastos cardíacos, uma vez que estas células contêm o código genético responsável por iniciar a síntese de colágeno fibrilar ${ }^{30}$. Na verdade, parece que os miofibroblastos têm importante função nesta síntese de colágeno ${ }^{7}$.

A hipertrofia dos miócitos cardíacos contribui para o aumento da massa miocárdica e/ou enrijecimento de suas paredes. A área transversal média dos miócitos pode dobrar, 
provocando mudança na forma desta célula ${ }^{29}$. Os resultados atuais demonstram aumento significativo da área transversal média dos miócitos nos animais que receberam LName. Houve também aumento tanto da área transversal média quanto da densidade numérica dos miócitos nos animais dos grupos controle com 25 e 40 dias. É importante salientar que, embora o crescimento do miocárdio adulto seja realizado por hipertrofia, há, até um certo período da vida pós-natal, um aumento hiperplástico cujos níveis vão gradativamente diminuindo com a idade até desaparecer. No rato, o número de miócitos quadruplica em dois anos de ida$\mathrm{de}^{31}$. Além disso, com o crescimento corporal, há um aumento no volume ventricular e conseqüente aumento na tensão mecânica das paredes do ventrículo, o que é um estímulo importante para o crescimento do miócito cardíaco. A observação do presente estudo nos animais dos grupos controle, provavelmente, se deve ao processo normal de crescimento dos animais no período estudado ${ }^{32}$. Pode também acompanhar a hipertrofia miocárdica a proliferação de células nãomiócitos. A proliferação destas células indica remodelamento estrutural do interstício cardíaco com aumento desproporcional das células não-miócitos, ocasionando perda da relação entre os compartimentos muscular e intersticial do miocárdio, o que é considerado patológico ${ }^{33}$.

Observou-se no presente estudo que, nos animais hipertensos com 25 dias de experimentação, o miocárdio ainda não apresentava alteração nas densidades de volume do interstício e do miócito, mas houve aumento significativo do peso cardíaco nesse período, o que parece ter sido causado pela hipertrofia dos miócitos cardíacos, uma vez que houve aumento significativo da área transversal média dos miócitos. Com 40 dias de experimentação, os animais hipertensos apresentaram miócitos com área transversal média um pouco maior, densidades de volume dos miócitos diminuída e do interstício significativamente aumentada. Estes resultados concordam com Contard e col ${ }^{34}$ que verificaram que o aumento do peso cardíaco em animais hipertensos experimentalmente está correlacionado, principalmente, com o aumento do conteúdo de colágeno no miocárdio hipertrofiado, também concorda com Zhang e col ${ }^{35}$, que afirmam que o tamanho do coração é, primariamente, determinado pelo diâmetro dos miócitos cardíacos nas fibras miocárdicas.

O modelo experimental de HA com L-Name causa uma fibrose (perivascular e intersticial reparativa) e desorganização do músculo cardíaco que são mais intensas que as observadas no modelo renovascular (Goldblatt II) ${ }^{4}$. Animais hipertensos pelo uso de L-Name e tratados com a administração de hidralazina, continuam apresentando remodelagem da microvascularização coronariana e hipertrofia miocárdica ${ }^{27}$. Estes resultados sugerem que as lesões miocárdicas em animais submetidos ao L-Name não são devidas exclusivamente a HA, mas estão associadas, principalmente, a inibição crônica da síntese do óxido nítrico e a lesão do endotélio vascular ${ }^{4,15,27}$.

Mudanças na túnica média do vaso são comuns na HA, enquanto que mudanças na túnica íntima são normal- mente associadas à hipercolesterolemia e aterosclerose e são restritas as grandes artérias. Há evidências, em modelos experimentais de HA, que a pressão sangüínea aumentada estimula a proliferação de células musculares lisas na túnica íntima dos vasos ${ }^{36}$. Essa proliferação pode ser o resultado de lesões endoteliais causadas por alta pressão intravascular ${ }^{37}$. O prejuízo da reserva vasodilatadora coronária na HA pode ser atribuído primariamente, às alterações estruturais nos vasos coronários de resistência. A hipertrofia miocárdica também pode contribuir para aumentar forças compressivas extravasculares que elevam a resistência coronária. A administração de L-Name por oito semanas foi associada a mudanças estruturais nos microvasos coronários, causando espessamento da túnica média e aumento de fibrose perivascular, mas essas alterações não ocorreram nas grandes artérias coronárias ${ }^{27}$. Os resultados atuais mostraram uma maior fibrose perivascular com o aumento no tempo de uso de L-Name em ratos. O aumento de tecido conjuntivo nas túnicas média e adventícia pode contribuir para a alteração na reatividade vasomotora e na reserva vasodilatadora coronária, levando, desse modo, a isquemia miocárdica com conseqüente perda de miócitos cardíacos ${ }^{38}$.

Os miócitos cardíacos hipertrofiados não têm tamanhos homogêneos nos estágios mais avançados da hipertrofia miocárdica, ocasionando também uma variação importante no espaço intercapilar ${ }^{39}$. Isto, adicionado ao processo inflamatório perivascular muito freqüente nestes casos, leva à isquemia de miócitos ${ }^{40}$. Apesar da possibilidade de haver proliferação de capilares miocárdicos, parece não ser suficiente para a manutenção dos níveis de oxigenação dos miócitos, havendo, em conseqüência, necrose de miócitos ${ }^{41}$. Na HA ocasionada pelo L-Name, o aumento da demanda metabólica miocárdica provocado pela HA está agravado porque os microvasos estão estreitados, há hipertensão e hipertrofia de miócitos, facilitando a isquemia miocárdica e provocando necrose de miócitos. Além do mais, a produção local de angiotensina II, endotelinas e/ou catecolaminas relacionadas à $\mathrm{HA}$, possivelmente, tem um papel importante na necrose miocárdica e subseqüente fibrose ${ }^{27}$. No presente estudo, a densidade numérica de miócitos nos animais hipertensos só diminuiu significativamente com 40 dias de uso do L-Name. Nesse período a isquemia miocárdica estava mais acentuada, tendo sido vistos inúmeros campos microscópicos com áreas de fibrose de substituição (região necrose antiga de miócitos) e outras áreas com infiltrado inflamatório (região de necrose recente de miócitos).

A apoptose é uma forma de morte celular distinta da necrose e pode ser explicada como um mecanismo homeostático que afeta a depleção seletiva de células cuja sobrevivência pode prejudicar o bem estar do organismo. A hipóxia relativa do miócito, devida à hipertrofia ventricular esquerda, pode também levar à apoptose. Parece que este processo de morte celular é um dos mecanismos que leva ao estágio final da doença cardíaca ${ }^{42,43}$. A proporção relativa de células necróticas/apoptóticas num determinado tecido depende do tipo e da duração da lesão. Necrose e apoptose de miócitos são observadas no miocárdio, inclusive no ho- 
mem. Sem dúvida, é a intensidade e a duração da isquemia miocárdica que ditam a ocorrência relativa de cada um dos tipos de morte celular. Estudos experimentais demonstraram que a isquemia miocárdica continuada provoca inicialmente morte celular por apoptose, seguida depois de algumas horas por necrose de miócitos ${ }^{44}$.

No presente estudo, a inibição da síntese do óxido nítrico concordou com os trabalhos que caracterizaram este modelo experimental na indução de HA. Com a estereologia, quantificaram-se as principais modificações adaptativas normais e patológicas, que ocorrem no miocárdio em decorrência do uso do L-Name como inibidor da síntese do óxido nítrico. Analisando a duração do uso de L-Name, constatou-se que há uma progressão nas lesões miocárdicas, inicialmente com hipertrofia dos miócitos e, mais tardiamente, com extensa fibrose intersticial e perivascular. Também tardiamente detectou-se diminuição de miócitos que, provavelmente, está associada aos fenômenos de necrose e apoptose.

\section{Agradecimentos}

Trabalho parcialmente financiado pelo CNPq (Proc. 57.41.14/97-1) e Faperj (Proc. E-26/170.315/95 e E-26/ $170.086 / 97)$

\section{Referências}

1. Leonetti G, Cuspidi C-The heart and vascular changes in hypertension. J Hypertension 1995; 13(suppl2): S29-S34.

2. Mandarim-de-Lacerda CA - Aspectos morfológicos do remodelamento ventricular esquerdo na cardiomiopatia hipertensiva. Arq Bras Cardiol 1995; 65: 523-7.

3. Chapman D, Weber KT, Egbali M - Regulation of fibrilar collagen types I and III and basement membrane type IV collagen gene expression in pressure overloaded rat myocardium. Circ Res 1990; 67: 787-94.

4. Moreno H, Metze K, Bento A - Chronic nitric oxide inhibition as a model oh hypertensive heart muscle disease. Basic Research Cardiol 1996; 91: 249-55.

5. Weber KT, Janicki JS, Pick R, Capasso JM, Anversa P- Myocardial fibrosis and pathologic hypertrophy in the rat with renovascular hypertension. Am J Cardiol 1990; 65: 1-7.

6. Wilke A, Funck R, Rupp H, Brilla CG - Effect of the renin-angiotensin-aldosterone system on the cardiac interstitium in heart failure. Basic Res Cardiol 1996 91(suppl 2): 79-84.

7. Weber KT - Metabolic responses of extracellular matrix in tissue repair. Ann Med 1997; 29: 333-8.

8. Banting JD, Wiseman SL, Adams MA-Hypertension without cardiac hypertrophy does not induce a cardiac baroreflex deficit. J Hypert 1995; 14: 1209-14.

9. Gardiner SM, Compton AM, Bennet T, Palmer RMJ, Moncada S - Regional haemodynamic changes during oral ingestion of NG-monomethyl-L-arginine or NG-nitro-L-arginine methyl ester in conscious Brattleboro rats. Br J Pharmacol 1990; 101: 10-2.

10. Ribeiro MO, Antunes E, De Nucci G, Lovisolo SM, Zatz R - Chronic inhibition of nitric oxide synthesis, a new model of arterial hypertension. Hypertension 1992; 20: 298-303.

11. Almeida PJ, Cabral AM, VasquezEC-O endotélio como modulador de respostas vasomotoras. Arq Bras Cardiol 1993; 60: 347-56.

12. Morton JJ, Beattie EC, Speirs A, Gulliver F - Persistent hypertension following inhibition of nitric oxide formation in the young Wistar rat: role of renin and vascular hypertrophy. J Hypertens 1993; 11: 1083-8.

13. Kristek F, Gerová M, Devát L, Varga I-Remodelling of septal branch of coronary artery and carotid artery in L-NAME treated rats. Physiol Res 1996; 45: 329-33.

14. Pereira, LMM, Mandarim-de-Lacerda CA - Morfologia del miocardio en ratas sometidas a inhibicion cronica de la sintesis de oxido nitrico. Rev Chil Anat 1996; 14: 147-55.

15. Mandarim-de-Lacerda CA, Pereira LMM - Stereology of the myocardium in hypertensive rats under chronic inhibition of nitric oxide synthesis. Biomed Res 1997; 8: 153-60.

16. Arnal JF, Armani EI, Chatellier G, Menard J, Michel JB - Cardiac weight in hypertension induced by nitric oxide synthase blockade. Hypertension 1993; 22 380-7.

17. Bernátová I, Pechanová O - NO-deficient hypertension induced by L-NAME treatment in rats. J Mol Cell Cardiol 1994; 26: 123-8.

18. Pereira LMM, Mandarim-de-Lacerda CA - Estereologia do miocárdio em ratos hipertensos pelo uso de inibidor da síntese do óxido nítrico. Rev Port Cardiol 1997; 16: 753-8.

19. Scherle W - A simple method for volumetry of organs in quantitative stereology. Mikroskopie 1970; 26: 57-60.

20. Mattfeldt T, Møbius HJ, Mall G - Orthogonal triplet probes: an efficient method for unbiased estimation of length and surface of objects with unknown orientation in space. J Microsc 1985; 139: 279-89.

21. Mandarim-de-Lacerda CA - Métodos quantitativos em morfologia. Rio de Janeiro, ed UERJ, 1995: 131

22. Gundersen HJG, Bagger P, Bendtsen TF et al - The new stereological tools: disector, fractionator, nucleator and point sampled intercepts and their use in pathological research and diagnosis. APMIS 1988; 96: 857-81.

23. Cruz-Orive LM, Weibel ER. Recent stereological methods for cell biology: a brief survey. Am J Physiol 1990;258 (Lung Cell Mol Physiol 2): L148-L156.

24. Gundersen HJG - Notes on the estimation of the numerical density of arbitrary profiles: the edge effect. J Microsc 1977; 11:219-23.

25. Zar JH - Biostatistical analysis. Upper Saddle River: Prentice-Hall, 1996: 662.

26. Sládek T, Gerová M,Znojil V, Devát L-Morphometric characteristics of cardiac hypertrophy induced by long-term inhibition of NO Synthase. Physiol Res 1996; 45: 335-8.

27. Numaguchi K, Egashira K, Takemoto Met al-Chronic inhibition of nitric oxide synthesis cause coronary microvascular remodeling in rats. Hypertension 1995; 26 [part 1]: 957-62.

28. Rakusan K, Korecky B, Mezl V - Cardiac hypertrophy and/or hyperplasia. Perspect Cardiovasc Res 1983; 7: 103-9.

29. Anversa P, Loud AV, Giacomelli F, Wiener J - Absolute morphometric study of myocardial hypertrophy in experimental hypertension II. Ultrastructure of myocytes and interstitium. Lab Invest 1978; 40: 341-9.

30. Weber KT, Clark WA, Janicki JS, Shroff SG - Physiological versus pathologic hypertrophy and the pressure overloaded myocardium. JCardiovasc Pharmacol 1987; 10: 537-50

31. Sasaki R, Watanabe Y, Morishita T - Estimation of the cell number of the heart muscle in normal rats. Tohoku J Exp Med 1968; 95: 177-84.

32. Rakusan K-Cardiac growth, naturatin and ageing. In: Zak R, Growth of the heart in health and disease. New York; Raven Press 1984; 131-64.

33. Weber KT, Brilla CG - Pathological hypertrophy and cardiac interstitium. Circulation 1991; 83: 1849-65.

34. Contard F, Koteliansky V, Marotte F, Dubus I, Rappaport L, Samuel JL-Specific alteration in the distributiun of extracellular matrix components within rat myocardium during the development of pressure overload. Lab Invest 1991; 64: 6575.

35. Zhang L, Summers KM, West MJ - Analysis of linkage of the ACE locus with measures of cardiac hypertrophy in the spontaneosly hypertensive rat. Clin Exp Pharmacol Physiol 1996; 23: 597-9.

36. Robinson TF, Cohen LG, Factor SM, Eghbali M, Blumenfield O-Structure and fuction of connective tissue in cardiac muscle:collagen type I and type III in endomysial struts and pericellular fibers. Scanning Microsc 1988; 2: 1005-15.

37. Caulfield JB, Borg TK - The collagen network of the heart. Lab Invest 1979; 40: 364-72.

38. Driss AB, Benessiano J, Poitevin P, Levy BI, and Michel JB - Arterial expansive remodeling induced by high flow rates. Am J Physiol 1997; 272: H851-H858.

39. Roberts JT - Arteries, veins and lymphatic vessels of the heart. In: Luisada AA, Development and structure of the cardiovascular system. New York; McGrawHill 1961: 85-118.

40. Okoshi MP, Matsubara LS, Franco M, Cicogna AC, Matsubara BB - Myocyte necrosis is the basis for fibrosis in renovascular hypertensive rats. Braz J Med Biol Res 1997; 30: 1135-44.

41. Suzuki Y, Harada K, Kawamura K, Matsuda H, Tarada G - Limited adaptation in chronically hypertrophied hearts from aortic constricted rats: increased inhomogeneity in cross sectional area of cardiomyocytes and intercapillary distance. Tohoku J Exp Med 1993; 170: 181-95.

42. Bing OHL - Hypothesis: apoptosis may be a mechanism for the transition to heart failure with chronic pressure overload. J Mol Cell Cardiol 1994; 26: 943-48.

43. Narula J, Haider N, Virmani R et al - Apoptosis in myocytes in end stage heart failure. N Eng J Med 1996; 335: 1182-9.

44. Fliss H, Gattinger D- Apoptosis in ischemic and reperfused rat myocardium. Circ Res 1996; 79: 949-56. 